\title{
Simultaneous uniportal video-assisted thoracic surgery for pulmonary nodules and synchronous mediastinal lesions
}

\author{
Liping Zeng, Runzhou Zhuang, Zhengliang Tu \\ Department of Thoracic Surgery, The First Affiliated Hospital, Zhejiang University, School of Medicine, Hangzhou, China \\ Videosurgery Miniinv 2021; 16 (2): 390-396 \\ DOI: https://doi.org/10.5114/wiitm.2020.101240
}

\begin{abstract}
Introduction: Video-assisted thoracic surgery (VATS) has been widely accepted in the diagnosis and treatment of thoracic diseases for the past three decades due to its small incision, minimal trauma, and rapid recovery after surgery. A growing number of patients with concurrent pulmonary nodules and mediastinal lesions have been detected. Simultaneous ipsilateral resection of coexisting lesions is a preferred procedure.

Aim: To introduce our technique and preliminary experience in performing uniportal video-assisted thoracic surgery (VATS) for the simultaneous resection of pulmonary and mediastinal lesions.

Material and methods: We retrospectively analysed 8 consecutive patients who underwent simultaneous uniportal VATS resection of coexisting lesions of the lung and mediastinum in our centre. The clinical data were recorded and analysed.

Results: A total of 8 patients were enrolled, and all patients successfully underwent surgery through a single incision; no perioperative deaths occurred. The average single incision length was $3.9 \pm 0.3 \mathrm{~cm}$, the operative time was 102.3 $\pm 54.4 \mathrm{~min}$, and the bleeding volume was $27.5 \pm 17.9 \mathrm{ml}$. The thoracic drainage time was $3.0 \pm 2.3$ days, with a mean volume of $390.6 \pm 361.3 \mathrm{ml}$. The length of postoperative hospital stay was $4.0 \pm 1.9$ (range: $3-9$ ) days. No serious complications were observed in the hospital or during postoperative follow-up.

Conclusions: Uniportal VATS is feasible and safe for the simultaneous resection of pulmonary and mediastinal lesions in selected patients and offers cosmetically pleasing incisions and promising clinical application prospects.
\end{abstract}

Key words: uniportal video-assisted thoracic surgery, simultaneous resection, pulmonary nodule, mediastinal lesion.

\section{Introduction}

Video-assisted thoracic surgery (VATS) has been widely accepted in the diagnosis and treatment of thoracic diseases for the past three decades due to its small incision, minimal trauma, and rapid recovery after surgery. With the revolution of thoracoscopic instruments, development of surgical techniques, and renewal of minimally invasive concepts, the number of ports needed for VATS has been gradually reduced from three or four to only one. Currently, uniportal VATS has been routinely applied in lobectomy, segmentecto- my, extended thymectomy, and more [1-3]. Compared with conventional VATS, uniportal VATS is cosmetically superior and could reduce postoperative pain.

Although the incidence of synchronous pulmonary nodules and mediastinal lesions remains relatively low, a growing number of these patients have been detected owing to widespread use of low-dose computed tomography (CT) screening. For these patients, staged surgery is a feasible option, albeit leading to more chronic incisional pain, poor cosmetic outcomes, and high medical costs. It is likely that a two-stage surgery could delay treatment for the second thoracic

\section{Address for correspondence}

Zhengliang Tu, Department of Thoracic Surgery, The First Affiliated Hospital, Zhejiang University School of Medicine, Hangzhou, China, e-mail: drtuzhengliang@zju.edu.cn 
tumour and may increase the surgical difficulty because of iatrogenic pleural adhesions. One-stage surgery could overcome these limitations and alleviate psychological discomfort. Reports concerning simultaneous resection with the VATS technique are scarce, especially with the uniportal approach [4-7]. Thus, it is worth discussing how to perform one-stage surgery for the treatment of coexisting ipsilateral pulmonary nodules and mediastinal lesions.

In the present study, we introduced an approach for simultaneous resection of the pulmonary nodules and mediastinal lesions by uniportal VATS. Altogether, 8 patients who presented with pulmonary nodules and mediastinal lesions underwent this operation, and the clinical data were retrospectively collected and analysed.

\section{Aim}

In the present study, we aimed to introduce an approach for simultaneous resection of the pulmonary nodules and mediastinal lesions by uniportal VATS. Altogether, 8 patients who presented with pulmonary nodules and mediastinal lesions underwent this operation, and the clinical data were retrospectively collected and analysed.

\section{Material and methods}

\section{General information}

Patients with pulmonary nodules and synchronous mediastinal lesions admitted to the Depart- ment of Thoracic Surgery in our hospital from January 2018 to April 2019 were enrolled. Data including the clinical characteristics, surgical details, and postoperative pathology results were retrospectively reviewed. All patients had routine preoperative evaluations, and the study was approved by the Ethics Committee of the First Affiliated Hospital, School of Medicine, Zhejiang University. The characteristics of the 8 patients, including the incision length, operation time, operative bleeding volume, postoperative thoracic drainage time, and complications, were retrospectively reviewed.

\section{Surgical method}

General anaesthesia was applied with double-lumen endotracheal intubation. Generally, resection of the pulmonary nodule was performed first, and the patient was placed in the lateral decubitus position (Photo $1 \mathrm{~A}$, position 1). A single $3-4 \mathrm{~cm}$ incision was made in the fourth intercostal space between the anterior and middle axillary lines. The incision was retracted with a wound protector. The surgeon stood on the ventral side, with the first assistant standing on the opposite side and $\mathrm{a} 30^{\circ}$ thoracoscope fixed on the posterior side of the incision (Photo $1 \mathrm{~B}$ ). For uniportal right upper lobectomy, the anterior pulmonary trunks were divided first, followed by the bronchus, posterior ascending artery, pulmonary vein, and fissure. For uniportal right lower lobectomy, our preferred sequence was the fissure, vein, artery, and bronchus. With regard to segmentectomy, preoperative $3 \mathrm{D}$ lung reconstruction was recommend-
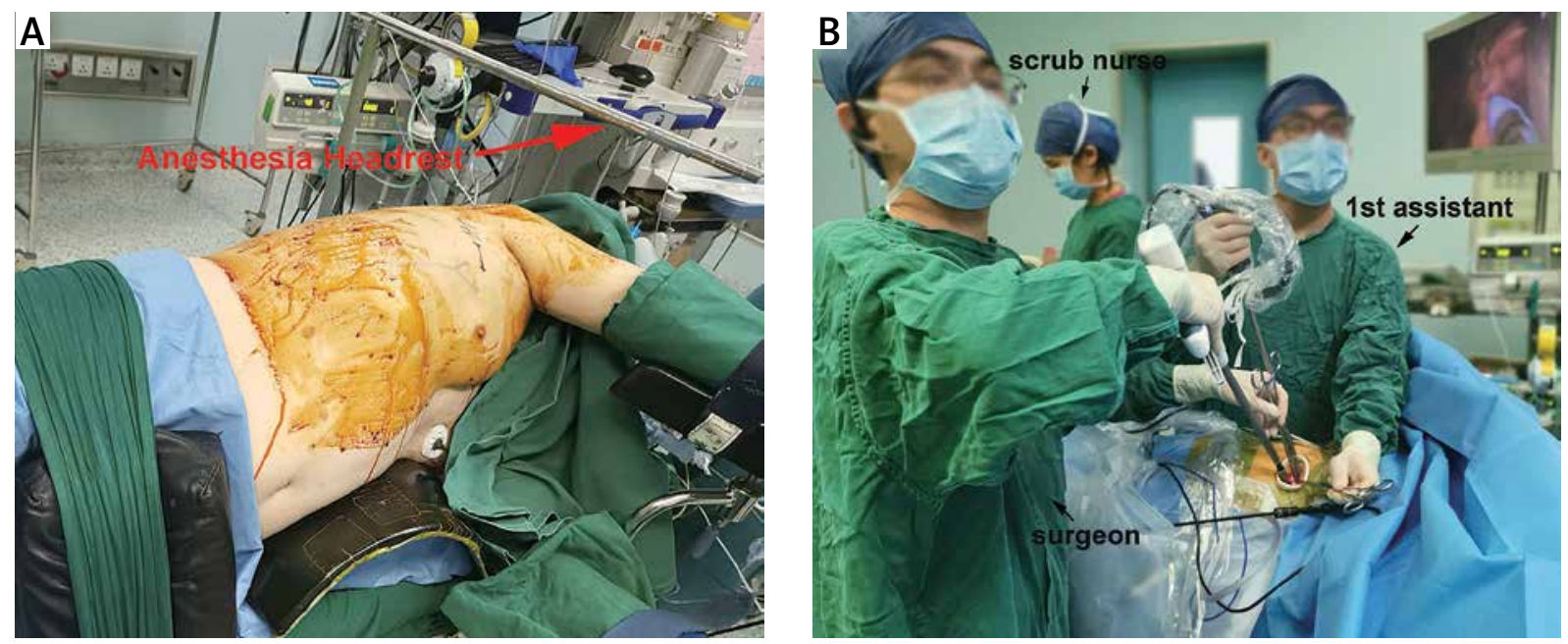

Photo 1. A - The patient was placed in the lateral decubitus position. B - The operating room setup and positions of the surgeon and the first assistant for resection of the pulmonary nodule 
ed for operative planning. The inflation-deflation method was applied for intersegmental borders, and intersegmental veins were precisely preserved. After the pulmonary nodule was resected (including mediastinal lymph node dissection for lung cancer), the patient was adjusted to an approximately $45^{\circ}$ semi-supine position (Photo $2 \mathrm{~A}$, position 2) by rolling the operating table, to excise the anterior mediastinal tumour. The surgeon and the first assistant stood on the dorsal side of the patient, facing the anterior mediastinum (Photos $2 \mathrm{~A}, \mathrm{~B}$ ). For posterior mediastinal tumours and most of the left anterior mediastinal tumours, the change in patient position was not always necessary. We preferred to dissect the mediastinal pleura around the tumour by using a curved suction tube and harmonic scalpel. The tumour was resected en bloc or biopsied. For thymoma, total thymectomy was performed. The recurrent laryngeal nerve and phrenic nerve should be well identified and protected. The specimen was retrieved using a glove through the incision, and a $22 \mathrm{~F}$ chest tube was placed.

All patients were followed up for 13 to 20 months, with a median follow-up of 15.4 months. Follow-up data were obtained through outpatient visits, telephone interviews, etc.

\section{Results}

From January 2018 to April 2019, 8 consecutive patients were enrolled and analysed, as shown in Ta- ble I. There were 3 male and 5 female patients, with an average age of $58.0 \pm 9.2$ (range: $47-71$ ) years. All patients underwent simultaneous resection of the pulmonary nodules and mediastinal lesions through a single incision. All mediastinal lesions were coincidentally located in the anterior mediastinum, and the median diameter was $2.8 \pm 1.7$ (range: $1.0-7.0$ ) cm. Multiple pulmonary nodules were detected and resected in 5/8 (62.5\%) cases, and the size of each pulmonary nodule was $\leq 1.5 \mathrm{~cm}$.

The surgery was successfully performed in all $8 \mathrm{pa}-$ tients, and the surgical details were shown in Table II. All surgeries were performed through a single incision in the fourth intercostal space, including two surgeries that were performed with the left transthoracic approach and six with the right transthoracic approach. The average incision length was 3.9 $\pm 0.3 \mathrm{~cm}$, with no conversion to multiport VATS or an open technique. For right pulmonary nodules coexisting with the anterior mediastinal lesion, the patients' position was switched from position 1 (Photo $1 \mathrm{~A}$ ) to 2 (Photo $2 \mathrm{~A}$ ) for resection of the pulmonary nodules and the mediastinal lesion. In the remaining 2 cases, the lateral decubitus position was applied throughout the surgery. Several types of pulmonary surgery were performed, including wedge resection (4 cases), segmentectomy (2 cases), and lobectomy ( 2 cases). Cyst excision ( 3 cases), limited thymectomy (4 cases), and total thymectomy (1 case) were selected according to the clinical diagnosis of the mediastinal lesions. The operative time was 102.3
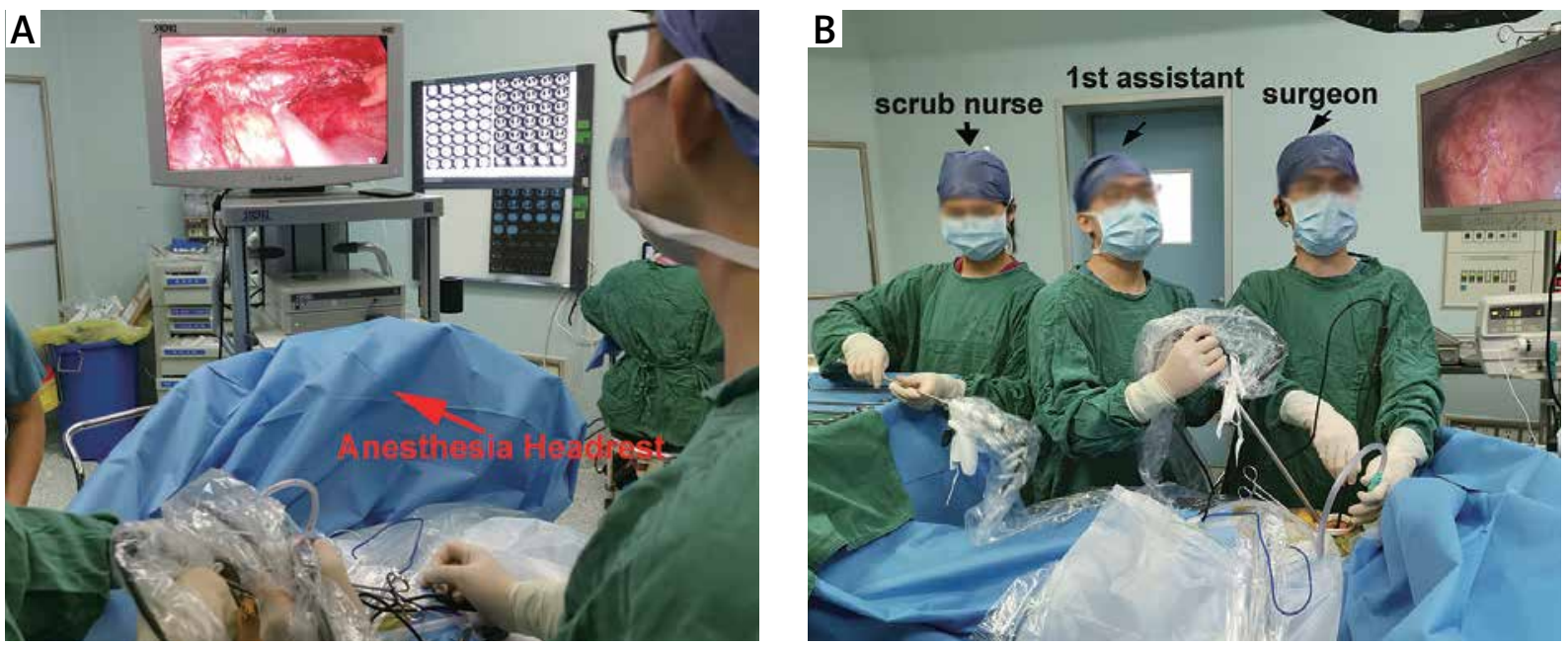

Photo 2. A - The patient was adjusted to an approximately $45^{\circ}$ semi-supine position by rolling the operating table. B - The operating room setup and positions of the surgeon and the first assistant for resection of the mediastinal lesion 
Table I. Patient demographics and clinical characteristics

\begin{tabular}{|lcccccc|}
\hline \multirow{2}{*}{ No. } & Gender & Age & \multicolumn{2}{c}{ Pulmonary nodule } & \multicolumn{2}{c|}{ Mediastinal lesion } \\
\cline { 3 - 7 } & & & Location (number of the nodule) & Size $[\mathrm{cm}]$ & Location & Size [cm] \\
\hline 1 & Female & 63 & RLL (1) & 1.5 & Anterior & 3 \\
\hline 2 & Male & 71 & RS8 (1), RS6 (1), RS2 (2) & $1.5,0.8,0.6,0.5$ & Anterior & 2.4 \\
\hline 3 & Female & 55 & Left lungs (disseminated) & Range: $2-4 \mathrm{~mm}$ & Anterior & 7 \\
\hline 4 & Female & 47 & RUL (1), RLL (1) & $0.5,0.7$ & Anterior & 2.2 \\
\hline 5 & Female & 51 & RS6 (1) & 0.8 & Anterior & 1 \\
\hline 6 & Female & 67 & RML (2) & $0.5,0.3$ & Anterior & 1.8 \\
\hline 7 & Male & 45 & LUL (1) & 0.5 & Anterior & 1.9 \\
\hline 8 & Male & 65 & RUL (3) & $0.9,0.5,0.4$ & Anterior & 3.3 \\
\hline
\end{tabular}

$R L L$ - right upper lobe, RS8 - the anterior basal segment of the right lower lobe, RS6 - the apical segment of the right lower lobe, RS2 - the posterior segment of the right upper lobe, $R U L$-right upper lobe, $R M L$ - right middle lobe, LUL - left upper lobe.

\pm 54.4 (range: $43-208$ ) $\mathrm{min}$, and the bleeding volume was $27.5 \pm 17.9$ (range: 10-50) $\mathrm{ml}$. The postoperative thoracic drainage time was $3.0 \pm 2.3$ (range: $2-9$ ) days, and the thoracic drainage volume was 390.6 \pm 361.3 (range: $60-1245$ ) $\mathrm{ml}$. The length of postoperative hospital stay was $4.0 \pm 1.9$ (range: $3-9$ ) days. Prolonged lung leakage occurred in 1 patient. There were no serious complications, such as myasthenia crisis, haemorrhage, respiratory failure, or perioperative death.

The postoperative pathology and follow-up details were shown in Table III. Five patients had multiple pulmonary nodes resected and pathologically diagnosed, including 3 with multiple lung adenocarcinomas and 1 with disseminated metastatic B-cell lymphoblastic lymphoma. One patient with thymoma, 6 patients with benign cysts, and 1 patient with B-cell lymphoblastic lymphoma were diagnosed with mediastinal lesions. No deaths or serious complications occurred during postoperative follow-up (1320 months, mean: 15.4 months).

\section{Discussion}

As the most prevalent malignancy, lung cancer is the leading cause of cancer-related mortality worldwide in both men and women [8]. Mediastinal lesions include thymoma, benign cyst, and schwannoma. The prevalence of incidental anterior mediastinal lesions is $0.73 \%$ in asymptomatic subjects [9]. Although thymoma is associated with increased risk of a second neoplasm, the incidence of pulmonary nodules synchronous with mediastinal lesions is relatively low. The mechanism of this association has not been validated. The demand for simultaneous resection of pulmonary nodules and mediastinal lesions is high to minimise surgical trauma, patient anxiety, and hospitalisation costs. However, reports concerning simultaneous resection are generally limited to case reports or case series with small samples, and management remains a challenge. Anatomically, it is feasible to resect the coexisting ipsilateral lesions through the same incision. VATS has been applied widely in the diagnosis and treatment of thoracic diseases. Uniportal, biportal, and triportal VATS were reported to be candidate approaches $[6,7]$. Uniportal VATS was first introduced in 2004 by Rocco [10]. Due to its advantages in minimising the incision and postoperative pain, it has been gradually adopted and is now utilised for lobectomy, segmentectomy, and thymectomy $[1,11,12]$. For patients with synchronous lung nodules and mediastinal lesions, reports of concurrent resection using a uniportal approach are still scarce.

Compared with a previous study using conventional VATS with three ports, the uniportal approach was comparable in operative time, intraoperative bleeding volume, and incidence of complications [4]. Similarly, a newly published study also implies that the uniportal approach is not inferior for the simultaneous resection of lung and mediastinal lesions [6]. Compared to the intercostal approach, subxiphoid uniportal VATS possesses unique advantages, such as no intercostal nerve injury and simultaneous access to the bilateral pleural cavities. However, this 


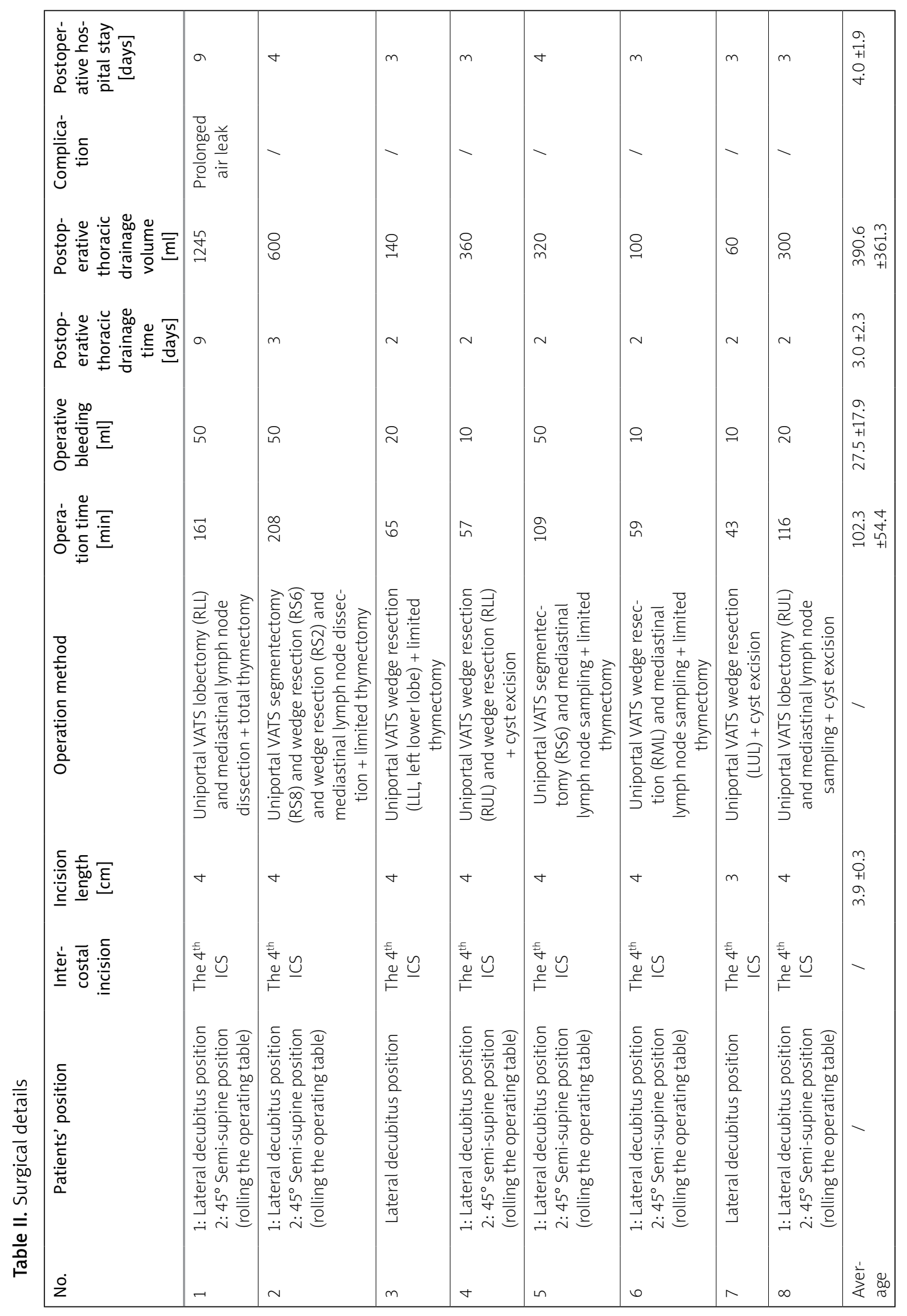


Table III. Pathology and follow-up

\begin{tabular}{|c|c|c|c|c|}
\hline \multirow[t]{2}{*}{ No. } & \multicolumn{2}{|l|}{ Pathological diagnosis } & \multirow{2}{*}{$\begin{array}{l}\text { Follow-up } \\
\text { [months] }\end{array}$} & \multirow[t]{2}{*}{ Status } \\
\hline & Pulmonary nodule & Mediastinal lesion & & \\
\hline 1 & Invasive adenocarcinoma (IA, T1bNOMO) & Thymoma (B2, Masaoka II) & 20 & Alive \\
\hline 2 & $\begin{array}{l}\text { Nodule1 (RS8): invasive adenocarcinoma, IA, T1bNOMO, } \\
\text { Nodule2 (RS2): adenocarcinoma in situ, IA, T1aNOMO, } \\
\text { Nodule3 (RS2): adenocarcinoma in situ, IA, T1aNOMO, } \\
\text { Nodule4 (RS6): pulmonary atypical hyperplasia }\end{array}$ & Bronchogenic cyst & 19 & Alive \\
\hline 3 & Metastatic B-cell lymphoblastic lymphoma & $\begin{array}{l}\text { B-cell lymphoblastic lym- } \\
\text { phoma }\end{array}$ & 16 & Alive \\
\hline 4 & $\begin{array}{l}\text { Nodule1 (RUL): chronic pulmonary inflammation, } \\
\text { Nodule2 (RLL): chronic pulmonary inflammation }\end{array}$ & Thymic cyst & 16 & Alive \\
\hline 5 & Invasive adenocarcinoma (IA, T1aNOMO) & Bronchogenic cyst & 13 & Alive \\
\hline 6 & $\begin{array}{l}\text { Nodule1 (RML): minimally invasive adenocarcinoma, IA, } \\
\text { T1aNOMO, Nodule2 (RML): minimally invasive adenocarcinoma, } \\
\text { IA, T1aNOMO }\end{array}$ & Bronchogenic cyst & 13 & Alive \\
\hline 7 & Pulmonary collagenous nodule & Bronchogenic cyst & 13 & Alive \\
\hline 8 & $\begin{array}{l}\text { Nodule1 (RUL): invasive adenocarcinoma, IA, T1aNOMO, } \\
\text { Nodule2 (RUL): adenocarcinoma in situ, IA, T1aNOMO, } \\
\text { Nodule3 (RUL): minimally invasive adenocarcinoma, IA, T1aNOMO }\end{array}$ & Thymic cyst & 13 & Alive \\
\hline
\end{tabular}

approach generally requires special extended equipment and is often difficult for left thoracic lesions because of the location of the heart. In addition, due to postural factors, the exposure of posterior mediastinal lesions and systemic mediastinal lymph node dissection of lung cancer is relatively tough. Additionally, adjustment of the patient's position is generally needed for simultaneous resection.

Among the 8 patients, 5 cases of adenocarcinoma were identified as pulmonary lesions, and 3/5 cases were of multiple primary lesions. Benign cysts were the most common histologic diagnosis for mediastinal lesions. In addition, this study included 1 case of suspected thymoma without a preoperative manifestation of myasthaenia gravis or signs of invasion in imaging. VATS appears to be feasible and safe for the resection of early-stage thymomas compared to median sternotomy $[13,14]$. Furthermore, VATS is reliable and safe for resection of large and invasive mediastinal tumours [15]. An increasing number of studies have reported the feasibility of uniportal VATS for mediastinal tumour resection and thymectomy, using either the left or right approach $[3,16,17]$. Therefore, uniportal VATS was selected in case no. 1 . The current follow-up showed no tumour recurrence. However, the therapeutic effect still needs to be further explored. In case no. 3,
CT-guided needle biopsy failed to confirm the pathological subtype; thus, uniportal VATS was selected for pathological diagnosis of the mediastinal lesion. Moreover, disseminated pleural implant metastasis was detected on the left lung surface during surgery, and wedge resection of the left lower lobe was also performed. Postoperative pathology revealed mediastinal B-cell lymphoblastic lymphoma accompanied by pleural metastasis. The underlying association between the coexisting pulmonary and mediastinal lesions needs to be clarified in largescale series.

For the simultaneous resection of pulmonary and mediastinal lesions, our experience is as follows: 1. for the sequence of pulmonary nodule and mediastinal tumour resection, we prefer to resect the pulmonary nodule first and then the mediastinal lesion, which mainly depends on the principle of management for malignant lesion and the surgeon's preference. 2. Appropriate incision selection is crucial for the success of surgery. The incision should be centred and suitable for the 2 main surgeries. Especially for anterior mediastinal tumours (all 8 cases of mediastinal lesions in this study were located in the anterior mediastinum), we suggest that the incision should be made slightly posteriorly (near the middle axillary line) to obtain a sufficient operation space. 
Generally, the patients need to be adjusted to an approximately $45^{\circ}$ semi-supine position by rolling the operating table to access the anterior mediastinal lesions. The camera should be placed at the ventral side of the incision. The surgeon and the first assistant should stand on the dorsal side of the patient, facing the anterior mediastinum. For left anterior mediastinal lesions and pulmonary nodules, the lateral decubitus position is most suitable. Of course, the semi-supine position would be an alternative choice, and this position would offer a better operative field for the resection of thymoma, depending on the surgeon's preference. 3. The indications for surgery should be strictly controlled. If the resection of multiple lesions is highly risky, concurrent surgery should be performed with extra caution because it could induce fatal complications. Finally, it should be noted that the patients should be secured well to the operating table to avoid falls when the table is rolled to change positions.

As long as the indications and contraindications of the surgery are strictly controlled, in the skilled hands of thoracic surgeons, simultaneous resection of pulmonary nodules and mediastinal lesions is feasible and safe. This approach could avoid the traditional secondary operation and greatly reduce trauma to the patient and minimise costs.

This study was limited because it was retrospective and had a small sample size.

\section{Conclusions}

Cases of pulmonary nodules associated with mediastinal tumours are relatively rare. For selected cases, uniportal VATS is feasible and safe for the diagnosis and treatment of pulmonary nodules and mediastinal lesions, leads to minimal surgical trauma and cosmetically pleasing incisions, and shows promising clinical application prospects. However, due to the small number of included cases and the selection bias of surgical patients, large-scale multicentre clinical trials are needed.

\section{Conflict of interest}

The authors declare no conflict of interest.

\section{References}

1. Hernandez-Arenas LA, Purmessur RD, Gonzalez-Rivas D. Uniportal video-assisted thoracoscopic segmentectomy. J Thorac Dis 2018; 10: S1205-14.
2. Sihoe AD. Uniportal video-assisted thoracic (VATS) lobectomy. Ann Cardiothorac Surg 2016; 5: 133-44.

3. Ooi A, Qiang F. Uniportal video assisted thoracoscopic surgery thymectomy (left approach). J Vis Surg 2016; 2: 12.

4. Lin F, Xiao Z, Mei J, et al. Simultaneous thoracoscopic resection for coexisting pulmonary and thymic lesions. J Thorac Dis 2015; 7: 1637-42.

5. Xie D, Xie H, Zhu Y, Jiang G. Simultaneous video-assisted thoracoscopic surgery sleeve lobectomy and thymectomy. Interact Cardiovasc Thorac Surg 2014; 19: 313-4.

6. Deng J, She Y, Zhao M, et al. Simultaneously thoracoscopic resection of lung cancer and anterior mediastinal lesions by video-assisted thoracoscopic surgery. Ann Transl Med 2019; 7: 333.

7. Abu-Akar F, Gonzalez-Rivas D, Yang C, et al. Subxiphoid uniportal VATS for thymic and combined mediastinal and pulmonary resections - a two-year experience. Semin Thorac Cardiovasc Surg 2019; 31: 614-9.

8. Verghese C, Redko C, Fink B. Screening for lung cancer has limited effectiveness globally and distracts from much needed efforts to reduce the critical worldwide prevalence of smoking and related morbidity and mortality. J Glob Oncol 2018; 4: 1-7.

9. Yoon SH, Choi SH, Kang CH, Goo JM. Incidental anterior mediastinal nodular lesions on chest CT in asymptomatic subjects. J Thorac Oncol 2018; 13: 359-66.

10. Rocco G, Martin-Ucar A, Passera E. Uniportal VATS wedge pulmonary resections. Ann Thorac Surg 2004; 77: 726-8.

11. Hirai K, Usuda J. Uniportal video-assisted thoracic surgery reduced the occurrence of post-thoracotomy pain syndrome after lobectomy for lung cancer. J Thorac Dis 2019; 11: 3896-902.

12. Scarci M, Pardolesi A, Solli P. Uniportal video-assisted thoracic surgery thymectomy. Ann Cardiothorac Surg 2015; 4: 567-70.

13. Qian LQ, Chen XO, Huang J, et al. A comparison of three approaches for the treatment of early-stage thymomas: robot-assisted thoracic surgery, video-assisted thoracic surgery, and median sternotomy. J Thoracic Dis 2017; 9: 1997-2005.

14. Li JF, Hui BG, Li X, et al. Video-assisted thoracic surgery for thymoma: long-term follow-up results and prognostic factors-single-center experience of 150 cases. J Thoracic Dis 2018; 10: 291-7.

15. Fang Y, Qin Z. Video-assisted thoracoscopic surgery is safe and reliable for large and invasive primary mediastinal tumors. Videosurgery Miniinv 2020. doi:10.5114/wiitm.2020.94528.

16. Ooi A, Sibayan M. Uniportal video assisted thoracoscopic surgery thymectomy (right approach). J Vis Surg 2016; 2: 13.

17. Wu CY, Heish MJ, Wu CF. Single port VATS mediastinal tumor resection: Taiwan experience. Ann Cardiothorac Surg 2016; 5: 107-11.

Received: 13.09.2020, accepted: 24.10.2020. 